H. FUJIMOTO

KODAI MATH. J.

11 (1988), 47-63

\title{
FINITENESS OF SOME FAMILIES OF MEROMORPHIC MAPS
}

\author{
By Hirotaka Fujimoto
}

\section{Introduction.}

In [3], H. Cartan proved that there exist at most two distinct nonconstant meromorphic functions on $C$ which have the same inverse images with multiplicities counted for three distinct values. Relating to this the author showed in his paper [5] that, for given $N+2$ hyperplanes $H_{1}, \cdots, H_{N+2}$ in $P^{N}(\boldsymbol{C})$ located in general position and effective divisors $E_{1}, \cdots, E_{N+2}$ on $C^{n}$, the set of all linearly nondegenerate meromorphic maps $f$ of $\boldsymbol{C}^{n}$ into $P^{N}(\boldsymbol{C})$ such that $f^{*} H_{i}=$ $E_{\imath}(1 \leqq i \leqq N+2)$ as divisors is finite. The purpose of this paper is to give a generalization of this result to the case of meromorphic maps of a compact complex manifold minus a thin analytic set into a projective algebraic manifold.

Let $Y$ be a projective algebraic manifold. For a complex holomorphic line bundle $L \rightarrow Y$ we denote the set of all holomorphic sections of $L$ by $H^{0}(Y, \mathcal{O}(L)$ ) and the set of all divisors $D_{\varphi}$ associated with zeros of nonzero holomorphic sections $\varphi$ of $L$ by $|L|$.

Definition 1.1. A meromorphic map $f$ of a complex manifold $X$ into $Y$ is said to be algebraically nondegenerate with respect to $L$ if $f(X) \not \subset \operatorname{Supp}\left(D_{\varphi}\right)$ for any $\varphi \in H^{0}\left(Y, O\left(L^{d}\right)\right)-\{0\}$, where $d$ is a positive integer.

The main result is stated as follows.

MaIN Theorem. Let $Y$ be an $N$-dimensional projective algebraic manifold, $L \rightarrow Y \quad$ a positive holomorphic line bundle and let $X$ be an n-dimensional compact complex manifold minus a thin analytic subset. Take effective divisors $E_{1}, \cdots, E_{N+2}$ on $X$ and $D_{1}, \cdots, D_{N+2} \in|L|$ such that

$$
\bigcap_{1 \leq j \leq N+2, j \neq i} \operatorname{Supp}\left(D_{j}\right)=\varnothing
$$

for each $i=1,2, \cdots, N+2$. Then the set $\mathcal{E}$ of all meromorphic maps of $X$ into $Y$ which are algebraically nondegenerate with respect to $L$ and satisfy the condition $f^{*}\left(D_{\imath}\right)=E_{\imath}(1 \leqq i \leqq N+2)$ is finite.

In the previous papers $([6],[7])$ the author stated that, for the particular case where $X=C^{n}$ or $X$ is a compact normal complex space minus an irreducible analytic set, the same conclusion holds under the weaker assumption that

Received September 4, 1987 
$D_{1}, \cdots, D_{\imath-1}, D_{i+1}, \cdots, D_{N+2}$ are algebraically independent with respect to $L$ for each $i=1,2, \cdots, N+2$. However, he found a gap in the proof of Lemma 4.3 in [6]. It is an open problem whether the assumption (1.2) of Main Theorem can be replaced by this weaker one or not.

In Main Theorem, we can take $E_{1}=\cdots=E_{N+2}=0$. Then we have

COROLLARY 1.3. Under the same assumption as in Main Theorem, the set of all meromorphic maps of $X$ into $Y-\bigcup_{1 \leqq i \geqq N+2} \operatorname{Supp} D_{\imath}$ which are algebraically nondegenerate with respect to $L$ is finite.

This is closely related to the result of Langmann [10].

\section{Preliminaries.}

Let $X, Y$ be ( $\sigma$-compact connected) complex manifolds and $f: X \rightarrow Y$ be a meromorphic map, namely, a many-valued map of $X$ into $Y$ such that (i) the graph $G^{f}=\{(x, y) ; y \in f(x)\}$ is an analytic subset of $X \times Y$, (ii) the projection $\pi_{X} \mid G^{f}: G^{f} \rightarrow X$ is proper and (iii) $f$ is single-valued on a nonempty open set $U$ in $X$. We denote by $I_{f}$ the set of all $x \in X$ such that $f(x)$ contains at least two points. Then, $I_{f}$ is an analytic set in $X$ with $\operatorname{codim} I_{f} \geqq 2$ and $f$ may be considered a single-valued map on $X-I_{f}$.

We consider particularly meromorphic maps into $P^{N}(\boldsymbol{C})$. Taking homogeneous coordinates $\left(w_{1}: \cdots: w_{N+1}\right)$ on $P^{N}(\boldsymbol{C})$, we set $H_{N+1}=\left\{w_{N+1}=0\right\}$. By identifying a point $\left(z_{1}, \cdots, z_{N}\right)$ in $\boldsymbol{C}^{N}$ with $\left(z_{1}: \cdots: z_{N}: 1\right)$ in $P^{N}(\boldsymbol{C})$, we may regard as $P^{N}(\boldsymbol{C})=\boldsymbol{C}^{N} \cup H_{N+1}$. We can show easily the following:

(2.1) Every meromorphic map $f: X \rightarrow P^{N}(C)$ with $f(X) \not \subset H_{N+1}$ can be written as

$$
f(x)=\left(\varphi_{1}(x): \cdots: \varphi_{N}(x): 1\right)
$$

outside a thin analytic set with meromorphic functions $\varphi_{1}, \cdots, \varphi_{N}$ on $X$. Conversely, each system of meromorphic functions $\varphi_{1}, \cdots, \varphi_{N}$ on $X$ gives a meromorphic map $f: X \rightarrow P^{N}(C)$ satisfying the identity (*).

We now consider the set $\mathscr{V}(X)$ of all one-codimensional irreducible analytic subsets of $X$.

Definition 2.2. We define a divisor $D$ on $X$ to be a map $D: \mathscr{V}(X) \rightarrow \boldsymbol{Z}$ which satisfies the condition that each $x \in X$ has a neighborhood $U$ such that

$$
\#\{V \in \mathscr{V}(X) ; U \cap V \neq \varnothing, D(V) \neq 0\}<+\infty,
$$

where $Z$ denotes the ring of all integers and $\# A$ means the number of elements in a set $A$.

For a divisor $D$ on $X$ we set $\mathscr{V}_{D}=\{V ; D(V) \neq 0\}$. The support of $D$ is 
defined by $\operatorname{Supp} D=\cup_{V \in \mathscr{V}_{D}} V$. The set $\mathscr{V}_{D}$ is at most countable. By notation $D=\sum_{i} m_{i} V_{\imath}$ we mean that $\mathscr{V}_{D} \subset\left\{V_{\imath} ; i=1,2, \cdots\right\}$ and $m_{i}=D\left(V_{\imath}\right)$, and we write $D=0$ if $\mathscr{V}_{D}=\varnothing$. A divisor $D$ is called effective if $D\left(V_{\imath}\right) \geqq 0$ for each $i$. For a divisor $D=\sum_{i} m_{i} V_{\imath}$ and an open subset $U$ of $X$ let each $V_{i} \cap U$ have the irreducible decomposition $V_{i} \cap U=\cup_{j} V_{\imath \jmath}$. Then we define the restriction of $D$ to $U$ by $D \mid U=\Sigma_{\imath, j} m_{i} V_{\imath \jmath}$.

Let $\varphi$ be a nonzero holomorphic function on a connected open subset $U$ of $X$. For each $x \in U$, taking holomorphic local coordinates $z$ with $x=(0)$, we expand $\varphi$ as

$$
\varphi(z)=\sum_{m=0}^{\infty} P_{m}(z)
$$

around $x$, where $P_{m}(z)$ is a homogeneous polynomial of degree $m$ or vanishes identically. We set

$$
\nu_{\varphi}(x):=\min \left\{m ; P_{m} \neq 0\right\},
$$

which does not depend on the choice of holomorphic local coordinates $z$. Set $Z=\{x \in U ; \varphi(x)=0\}$ and consider the irreducible decomposition $Z=\cup_{\imath} Z_{\imath}$. Then, $\nu_{\varphi}(x)$ is equal to a constant $m_{\imath}$ on each $R(Z) \cap Z_{\imath}$, where $R(Z)$ denotes the set of all regularities of $Z$. We define the zero divisor of $\varphi$ by $D_{\varphi}:=\sum_{i} m_{\imath} Z_{\imath}$. Let $f$ be a nonzero meromorphic function on $X$. For each $x \in X$, taking nonzero holomorphic functions $\varphi$ and $\phi$ on a neighborhood of $x$ with $f=\varphi / \phi$, we define the order of $f$ at $x$ by $\nu_{f}:=\nu_{\varphi}-\nu_{\psi}$. It is easily seen that there exists exactly one divisor $D_{f}=\sum_{i} m_{i} V_{\imath}$ on $X$ such that $\nu_{f}(x)=0$ on $X-\operatorname{Supp} D_{f}$ and $\nu_{f}(x)=m_{\imath}$ on $V_{i} \cap R\left(\operatorname{Supp} D_{f}\right)$. We call $\operatorname{ord}_{V}(f):=D_{f}(V)$ the order of $f$ along $V$ for each $V \in \mathscr{V}(X)$. The zero divisor $Z_{f}$ and the pole divisor $P_{f}$ of $f$ are defined by $Z_{f}:=\sum_{m_{\imath}>0} m_{i} V_{\imath}$ and $P_{f}=\sum_{m_{i}<0}\left(-m_{\imath}\right) V_{\imath}$ respectively.

Proposition 2.3. For two nonzero meromorphic functions $f_{1}$ and $f_{2}$ on $X$ the following three conditions are mutually equivalent;

(i) there is a nowhere zero holomorphic function $h$ with $f_{2}=h f_{1}$,

(ii) $D_{f_{1}}=D_{f_{2}}$,

(iii) there exists an analytic set $A$ of pure codimension one such that $A \supset \operatorname{Supp} D_{f_{1}} \cup \operatorname{Supp} D_{f_{2}}$ and each irreducible component of $A$ contains at least one point $x \in R(A)$ with $\nu_{f_{1}}(x)=\nu_{f_{2}}(x)$.

Particularly, if $X$ is compact, the condition (i) can be replaced by

(i )' there exists a nonzero constant $c$ with $f_{2}=c f_{1}$.

Proof. It is obvious that (i) implies (ii) and (ii) implies (iii). Suppose that $f_{1}$ and $f_{2}$ satisfy the condition (iii), and set $h:=f_{1} / f_{2}$. Then,

$$
\operatorname{Supp} D_{h} \subset \operatorname{Supp} D_{f_{1}} \cup \operatorname{Supp} D_{f_{2}} \subset A \text {. }
$$

We can write $D_{h}=\sum_{i} m_{\imath} A_{\imath}$, where $m_{\imath}$ are integers and $A_{\imath}$ are irreducible components of $A$. By the assumption, for each $i$ there exists one point $x_{i} \in R(A) \cap A_{\imath}$ such that $\nu_{f_{1}}\left(x_{\imath}\right)=\nu_{f_{2}}\left(x_{\imath}\right)$. This implies that 


$$
m_{\imath}=\nu_{h}\left(x_{\imath}\right)=\nu_{f_{1}}\left(x_{\imath}\right)-\nu_{f_{2}}\left(x_{\imath}\right)=0
$$

for each $i$. Therefore, $D_{h}=0$. This means that $h$ is a nowhere zero holomorphic function on $X$ and so $f_{\imath}(i=1,2)$ satisfy the condition (i). Here, $h$ is constant if $X$ is compact.

Let $f: X \rightarrow Y$ be a meromorphic map and $D$ be a divisor on $Y$ such that $f(X) \not \subset \operatorname{Supp} D$. For each $x \in X-I_{f}$ we can take a neighborhood $U$ of $x$ in $X$ and a neighborhood $V$ of $f(x)$ such that $f(U) \subset V$ and $D \mid V=D_{\varphi}$ for a nonzero meromorphic function $\varphi$ on $V$. Obviously, $\varphi \circ f \mid U$ is a nonzero meromorphic function on $U$ and the divisor $D_{\varphi \circ f}$ does not depend on the choice of the above $\varphi$. Then, there exists exactly one divisor $D^{*}$ on $X-I_{f}$ such that $D^{*} \mid U=D_{\varphi \circ f}$ for each $\varphi \circ f$ with the above property. Let $D^{*}=\sum_{i} n_{i} V_{\imath}$ on $X-I_{f}$. Since $I_{f}$ is of codimension $\geqq 2, \bar{V}_{i} \in \mathscr{V}(X)$ and $\left\{\bar{V}_{\imath}\right\}$ is locally finite. We call the divisor $f^{*}(D):=\sum_{i} n_{\imath} \bar{V}_{\imath}$ the pull-back of $D$ by $f$.

\section{Langmann's finiteness theorem for nowhere zero holomorphic functions.}

For a complex manifold $X$ we denote the field of all meromorphic functions on $X$ by $M(X)$ and the multiplicative group of all nowhere zero holomorphic functions on $X$ by $H^{*}(X)$.

Let $\tilde{X}$ be a complex manifold and $X$ an open subset of $\tilde{X}$ such that $A:=\tilde{X}-X$ is a thin analytic set in $\tilde{X}$. Regarding $M(\tilde{X})$ and $H^{*}(X)$ as subsets of $M(X)$ naturally, we set $H_{\tilde{X}}^{*}(X):=H^{*}(X) \cap M(\tilde{X})$. The multiplicative group $C^{*}:=C-\{0\}$ may be considered as a subgroup of the group $H_{\tilde{X}}^{*}(X)$. We consider the factor group $G:=H_{\tilde{X}}^{*}(X) / C^{*}$. For each $h$ in $H_{\tilde{X}}^{*}(X)$ we denote by [h] the class in $G$ which contains $h$.

Proposition 3.1 (cf., [9], Satz 3.4). In the above situation, if $\tilde{X}$ is compact and $A$ has s irreducible components, then $\operatorname{rank}_{Z} G \leqq s-1$.

Proof. We may assume that each irreducible component $A_{t}(1 \leqq t \leqq s)$ of $A$ is of codimension one because every $h$ in $H_{\tilde{X}}^{*}(X)$ has no zero on $A_{t}-\left(\bigcup_{u \neq t} A_{u}\right)$ whenever $A_{t}$ is of codimension $\geqq 2$. We first consider the case $s=1$. For each $h$ in $H_{\tilde{X}}^{*}(X) h$ is holomorphic on $\tilde{X}$ if ord $_{A} h \geqq 0$, and $1 / h$ is holomorphic on $\tilde{X}$ if $\operatorname{ord}_{A} h<0$. In either case, $h$ is necessarily a constant by the maximum principle. This shows that $\operatorname{rank}_{Z} G=0$. Suppose that $s \geqq 2$. We define a $Z$-homomorphism of $G$ into $Z^{s-1}$ by

$$
\Phi(h)=\left(\operatorname{ord}_{A_{1}} h, \cdots, \operatorname{ord}_{A_{s-1}} h\right) \in Z^{s-1} \quad\left(h \in H_{\tilde{X}}^{*}(X)\right) .
$$

For $h_{1}$ and $h_{2}$ in $H_{\tilde{X}}^{*}(X)$, if $\Phi\left(h_{1}\right)=\Phi\left(h_{2}\right)$, the meromorphic function $\varphi:=h_{1} / h_{2}$ has neither zero nor pole on $\tilde{X}-A_{s}$. By the above argument, $\varphi$ is a constant 
and so $\left[h_{1}\right]=\left[h_{2}\right]$. Therefore, $\Phi$ is injective. The group $G$ may be considered as a subgroup of $Z^{s-1}$. We then have $\operatorname{rank}_{Z} G \leqq s-1$.

We now give the following finiteness theorem.

THEOREM 3.2. Let $\tilde{X}$ be a compact complex manifold and $X$ be an open subset of $\tilde{X}$ such that $A:=\tilde{X}-X$ is a thin analytic set in $\tilde{X}$. For nonzero meromorphic functions $\alpha_{\imath}(1 \leqq i \leqq p)$, consider the set $\mathscr{F}$ of all elements $\left(\left[h_{1}\right], \cdots,\left[h_{p}\right]\right)$ $\in G^{p}$ with $h_{i} \in H_{\tilde{X}}^{*}(X)$ which satisfy the conditions

$$
\sum_{\imath=1}^{p} \alpha_{i} h_{\imath}=1
$$

and $\sum_{i \in I} \alpha_{i} h_{\imath} \neq 0$ for any $I \subset\{1,2, \cdots, p\}$. Then, $\# \mathscr{F}$ is bounded by a constant $R(p, s)$ depending only on $p$ and the number $s$ of irreducible components of $A$.

This is a special case of Langmann [10], Lemma 1.2. We shall give here a function-theoretic direct proof, which provides a better estimate than his, particularly, in the case where $\alpha_{i} h_{\imath}(1 \leqq i \leqq p)$ are linearly independent over $\boldsymbol{C}$. For our purpose, we need some lemmas.

Let $U^{n}:=\left\{\left(z_{1}, \cdots, z_{n}\right) ;\left|z_{2}\right|<1\right\}$ and $A=\left\{z_{1}=0\right\} \cap U^{n}$.

LEMmA 3.3. If $V$ is a d-dimensional $\boldsymbol{C}$-vector space of $M\left(U^{n}\right)$, then

$$
\#\left\{\operatorname{ord}_{A} \varphi ; \varphi \in V-\{0\}\right\} \leqq d .
$$

Proof. Take a vector subspace $W$ of $V$ with $\operatorname{dim} W=d-1$. It suffices to show that

$$
\#\left\{\operatorname{ord}_{A} \varphi ; \varphi \in V-\{0\}\right\} \leqq \#\left\{\operatorname{ord}_{A} \varphi ; \varphi \in W-\{0\}\right\}+1,
$$

which gives Lemma 3.3 by induction on $d$. Assume that there exists some $\varphi_{0}$ in $V-\{0\}$ such that $\operatorname{ord}_{A} \varphi_{0} \notin\left\{\operatorname{ord}_{A} \varphi ; \varphi \in W-\{0\}\right\}$. Take any $\varphi \in V-\{0\}$ with $\operatorname{ord}_{A} \varphi \neq \operatorname{ord}_{A} \varphi_{0}$. Then, we can see $\varphi=c \varphi_{0}+\psi$ for some $c$ in $C$ and $\phi$ in $W-\{0\}$ and we easily see $\operatorname{ord}_{A} \varphi=\operatorname{ord}_{A} \psi \in\left\{\operatorname{ord}_{A} \chi ; \chi \in W-\{0\}\right\}$. This completes the proof.

LEMMA 3.4. Let $\alpha_{1}, \cdots, \alpha_{p} \in M\left(U^{n}\right)^{*}:=M\left(U^{n}\right)-\{0\}$ and $P$ a subset of $M\left(U^{n}\right)^{*}$ such that $[P]=\{[h] ; h \in P\}$ is a finitely generated subgroup of the factor group $M\left(U^{n}\right)^{*} / C^{*}$. Consider the set $\mathscr{G}_{p}$ of all elements $\left(\operatorname{ord}_{A} h_{1}, \cdots, \operatorname{ord}_{A} h_{p}\right) \in Z^{p}$ with $h_{\imath}$ in $P$ which satisfy the conditions

$$
\sum_{i=1}^{p} \alpha_{i} h_{\imath}=1, \quad \sum_{i \in I} \alpha_{i} h_{\imath} \neq 0
$$

for any $I \subset\{1, \cdots, p\}$. Then $\# \mathscr{G}_{p}$ is bounded by a constant depending only on $p$ and $r=\operatorname{rank}_{Z}[P]$.

Proof. Since $[P]$ is countable, we can find a point $a^{\prime}=\left(a_{2}, \cdots, a_{n}\right)$ with 
$\left|a_{\imath}\right|<1$ such that, setting $\alpha_{i}^{*}(z):=\alpha_{i}\left(z, a^{\prime}\right)$ and $h_{i}^{*}(z)=h_{i}\left(z, a^{\prime}\right)$ for $h_{1}, \cdots, h_{p} \in P$ satisfying the condition in Lemma 3.4, we have $\sum_{i \in I} \alpha_{\imath}^{*} h_{\imath}^{*} \neq 0$ for any $I \subset\{1, \cdots, p\}$ and $\operatorname{ord}_{0} \alpha_{i}^{*}=\operatorname{ord}_{A} \alpha_{\imath}, \operatorname{ord}_{0} h_{\imath}^{*}=\operatorname{ord}_{A} h_{2}$. Therefore, we may consider $\alpha_{i}^{*}$ and $h_{\imath}^{*}$ instead of $\alpha_{\imath}$ and $h_{\imath}$. By this reason, we assume $n=1$.

Let $h_{1}, \cdots, h_{p}$ satisfy the condition (3.5) and set $f_{\imath}=\alpha_{i} h_{\imath}(1 \leqq i \leqq p)$. We first consider systems $\left(f_{1}, \cdots, f_{p}\right)$ satisfying the additional condition that $f_{1}, \cdots, f_{p}$ are linearly independent over $\boldsymbol{C}$. By the assumption that

we have

$$
f_{1}+f_{2}+\cdots+f_{p}=1
$$

$$
\frac{f_{1}^{(l)}}{f_{1}} f_{1}+\frac{f_{2}^{(l)}}{f_{2}} f_{2}+\cdots+\frac{f_{p}^{(l)}}{f_{p}} f_{p}=0 \quad(1 \leqq l \leqq p-1),
$$

where $f_{\imath}^{(l)}$ denotes the $l$-th derivatives of $f_{\imath}$. Therefore,

$$
f_{\imath}=(-1)^{\imath-1} \frac{\operatorname{det}\left(\frac{f_{1}^{(l)}}{f_{1}}, \cdots, \frac{f_{l-1}^{(l)}}{f_{2-1}}, \frac{f_{i+1}^{(l)}}{f_{\imath+1}}, \cdots, \frac{f_{p}^{(l)}}{f_{p}} ; 1 \leqq l \leqq p-1\right)}{\operatorname{det}\left(\frac{f_{1}^{(l)}}{f_{1}}, \cdots, \frac{f_{p}^{(l)}}{f_{p}} ; 0 \leqq l \leqq p-1\right)} .
$$

We now take $g_{1}, \cdots, g_{r} \in M\left(U^{1}\right)$ which give a system of generators of $[P]$, where $r=\operatorname{rank}_{\boldsymbol{Z}}[P]$. Each $h_{\imath}$ can be written as

$$
h_{\imath}=c_{i} g_{1}^{m_{i}} \cdots g_{r}^{m_{2 r}}
$$

with some $c_{i} \in \boldsymbol{C}^{*}$ and $m_{\imath j} \in \boldsymbol{Z}$. Then,

$$
\left(\frac{f_{i}^{\prime}}{f_{\imath}}\right)^{(l)}=\left(\frac{\alpha_{2}^{\prime}}{\alpha_{\imath}}\right)^{(l)}+m_{i 1}\left(\frac{g_{1}^{\prime}}{g_{1}}\right)^{(l)}+\cdots+m_{\imath r}\left(\frac{g_{r}^{\prime}}{g_{r}}\right)^{(l)}
$$

for each $l$. On the other hand, for each $l$ there exists a polynomial $P_{l}\left(u_{1}, \cdots, u_{l}\right)$ such that

$$
\frac{f_{i}^{(l)}}{f_{\imath}}=P_{l}\left(\frac{f_{i}^{\prime}}{f_{\imath}},\left(\frac{f_{2}^{\prime}}{f_{\imath}}\right)^{\prime}, \cdots,\left(\frac{f_{i}^{\prime}}{f_{\imath}}\right)^{(l-1)}\right)
$$

and $P_{l}$ is isobaric of weight $l$ if we associate weight $k$ with each variable $u_{k}$, namely, if $P_{l}\left(u, u^{2}, \cdots, u^{l}\right)$ is homogeneous of degree $l$ as a polynomial in $u$. From these facts, we can conclude that both of the denominator $W_{1}$ and the numerator $W_{2}$ of the right hand side of (3.6) are written as polynomials of $\left(\alpha_{i}^{\prime} / \alpha_{\imath}\right)^{(l)}$ and $\left(g_{j}^{\prime} / g_{j}\right)^{(l)}(1 \leqq i \leqq p, 1 \leqq j \leqq r, 0 \leqq l \leqq p-2)$ which are isobaric of weight $p(p-1) / 2$ if we associate weight $l$ with each $\left(\alpha_{i}^{\prime} / \alpha_{\imath}\right)^{(l-1)}$ and $\left(g_{j}^{\prime} / g_{j}\right)^{(l-1)}$. Let $V$ be the set of all polynomials of $\left(\alpha_{i}^{\prime} / \alpha_{\imath}\right)^{(l)}$ and $\left(g_{j}^{\prime} / g_{j}\right)^{(l)}(l=0,1, \cdots, p-2)$ which are isobaric of weight $p(p-1) / 2$. Then, $V$ is a $C$-vector subspace of $M\left(U^{1}\right)$ with $\operatorname{dim} V \leqq d(r+p, p-1, p(p-1) / 2)$, where $d(u, v, w)$ denotes the dimension of the $C$-vector space of all polynomials of $u \times v$ variables $x_{i j}(1 \leqq i \leqq u$, $1 \leqq j \leqq v$ ) which are isobaric of weight $w$ if we associate weight $j$ with each $x_{\imath \jmath}$. In view of Lemma 3.3 , we have

$$
\#\left\{\operatorname{ord}_{A} \varphi ; \varphi \in V-\{0\}\right\} \leqq d(r+p, p-1, p(p-1) / 2) .
$$


This shows that the number of possible values of $\operatorname{ord}_{A} W_{1}$ and of $\operatorname{ord}_{A} W_{2}$ are both at most $d(r+p, p-1, p(p-1) / 2)$. Therefore, the number of possible values of each $\operatorname{ord}_{A} f_{2}$ is at most $d(r+p, p-1, p(p-1) / 2)^{2}$. Since $\operatorname{ord}_{A} h_{2}=\operatorname{ord}_{A} f_{i}-$ $\operatorname{ord}_{A} \alpha_{i}$, we conclude that

$$
\begin{aligned}
& \#\left\{\left(\operatorname{ord}_{A} h_{1}, \cdots, \operatorname{ord}_{A} h_{p}\right) \in \mathscr{G}_{p} ; \alpha_{i} h_{\imath}(1 \leqq \imath \leqq p) \text { are linearly independent }\right\} \\
& \leqq d(r+p, p-1, p(p-1) / 2)^{2 p} .
\end{aligned}
$$

We now start to prove Lemma 3.4 by induction on $p$. The case $p=1$ is trivial. Assume that Lemma 3.4 is true for the case $\leqq p-1$. Set $\mathscr{F}:=$ $\left\{\left(f_{1}, \cdots, f_{p}\right) ; f_{1}:=\alpha_{1} h_{1}, \cdots, f_{p}:=\alpha_{p} h_{p}\right.$ satisfy the condition (3.5)\}. For each subset $I$ of $\{1, \cdots, p\}$ we consider the set $\mathscr{F}_{I}$ of all elements $\left(f_{1}, \cdots, f_{p}\right)$ in $\mathscr{F}$ such that $f_{\imath}(i \in I)$ are linearly independent over $C$ and they satisfy the identity

$$
\sum_{\imath \in I} c_{\imath} f_{\imath}=1
$$

for some $c_{i} \in C^{*}(i \in I)$. Then, as is easily seen, $\mathscr{F}=\cup_{I} \mathscr{F}_{I}$. So, it suffices to show that

$$
\#\left\{\left(\operatorname{ord}_{A} f_{1}, \cdots, \operatorname{ord}_{A} f_{p}\right) ;\left(f_{1}, \cdots, f_{p}\right) \in \mathscr{F}_{I}\right\}
$$

is finite for an arbitrarily fixed $I$. Changing indices, we assume $I=\{1,2, \cdots, q\}$ $(1 \leqq q<p)$. We next consider a set $\mathscr{J}=\left(J_{q+1}, \cdots, J_{p}\right)$ of proper subsets of $\{1,2, \cdots, p\}$ such that $l \in J_{l}, J_{l} \cap\{1,2, \cdots, q\} \neq \varnothing$, and define the set $\mathscr{F}_{I}:=\bigcap_{l=1}^{p} \mathscr{F}_{I, J_{l}}$, where $\mathscr{F}_{I, J_{l}}$ is the set of all $\left(f_{l}\right) \in \mathscr{F}_{I}$ satisfying the condition that there exist some $d_{i} \in C^{*}$ such that $\sum_{i \in J_{l}} d_{2} f_{\imath}=0$ and $\sum_{i \in I^{\prime}} d_{\imath} f_{i} \neq 0$ for any $I^{\prime} \subsetneq J_{l}$. For an element $\left(f_{1}, \cdots, f_{p}\right) \in \mathscr{F}_{I}$ satisfying the identity (3.7), we have

$$
\left(1-c_{1}\right) f_{1}+\cdots+\left(1-c_{q}\right) f_{q}+\sum_{l=q+1}^{p} f_{l}=0
$$

for some $c_{\imath}$ in $C^{*}$. For each $l=q+1, \cdots, p$, if we take a minimal subset $J_{l}$ such that $l \in J_{l}$ and $\sum_{i \in J_{l}} d_{\imath} f_{l}=0$, then $J_{l}$ intersects with $\{1,2, \cdots, q\}$ by the condition (3.5), where $d_{i}=1-c_{\imath}$ for $1 \leqq i \leqq q$ and $d_{j}=1$ for $q+1 \leqq j \leqq p$. This shows that $\left(f_{i}\right)$ is contained in $\mathscr{F}_{1}^{\mathscr{f}}$ for $\mathscr{J}=\left(J_{l}\right)$. Therefore, $\mathscr{F}_{I}=\cup_{\mathscr{g}} \mathscr{F}_{1}^{\mathscr{f}}$. On the other hand, by the above shown facts we have

$$
\#\left\{\left(\operatorname{ord}_{A} f_{1}, \cdots, \operatorname{ord}_{A} f_{q}\right) ;\left(f_{1}, \cdots, f_{q}\right) \in \mathscr{F}_{I}\right\}<\infty .
$$

Moreover, for $J_{l}=\left\{j_{0}, j_{1}, \cdots, j_{s}\right\}$ with $1 \leqq j_{0} \leqq q$, by applying the induction hypothesis to the functions $f_{\jmath_{1}} / f_{\jmath_{0}}, \cdots, f_{\jmath_{s}} / f_{\jmath_{0}}$ we see

$$
\#\left\{\left(\operatorname{ord}_{A} f_{\partial_{1}}-\operatorname{ord}_{A} f_{J_{0}}, \cdots, \operatorname{ord}_{A} f_{\jmath_{s}}-\operatorname{ord}_{A} f_{J_{0}}\right) ;\left(f_{2}\right) \in \mathscr{F}_{I, J_{l}}\right\}<\infty .
$$

It then follows that

$$
\#\left\{\left(\operatorname{ord}_{A} f_{\jmath_{1}}, \cdots, \operatorname{ord}_{A} f_{\jmath_{q}}\right) ;\left(f_{\imath}\right) \in \mathscr{F}_{I, J_{l}}\right\}<\infty .
$$

Since $l \in J_{l}$ for any $l(q+1 \leqq l \leqq p)$, we conclude 


$$
\#\left\{\left(\operatorname{ord}_{A} f_{1}, \cdots, \operatorname{ord}_{A} f_{p}\right) ;\left(f_{\imath}\right) \in \mathscr{F}_{1}\right\}<\infty
$$

and so $\#\left\{\left(\operatorname{ord}_{A} f_{2}\right) ;\left(f_{2}\right) \in \mathscr{F}_{I}\right\}$ is finite. As is seen by the above arguments, $\# \mathscr{F}$ is bounded by a constant depending only on $p$ and $\operatorname{rank}_{z}[P]$. This completes the proof of Lemma 3.4.

Proof of Theorem 3.2. Let $A=\bigcup_{t=1}^{s} A_{t}$ be the irreducible decomposition of $A$. We may assume $\operatorname{codim} A_{t} \geqq 1$ for each $t$. For each $A_{t}$ we take a point $x_{t} \in R\left(A_{t}\right)$ and choose holomorphic local coordinates $\left(z_{1}^{(t)}, \cdots, z_{n}^{(t)}\right)$ on a neighborhood $U_{t}$ of $x_{t}$ with $x_{t}=(0)$ such that $U_{t}=\left\{\left|z_{\imath}^{(t)}\right|<1\right\}$ and $A \cap U_{t}=\left\{z_{1}^{(t)}=0\right\} \cap$ $U_{t}$. Set $P:=H_{\tilde{X}}^{*}(X)$, which may be considered as a subgroup of $M\left(U_{t}\right)^{*}$ because the restriction map of $M(\tilde{X})$ into $M\left(U_{t}\right)$ is injective. We may also regard $[P]:=\{[h] ; h \in P\}$ as a subset of $H_{U_{t}}^{*}\left(U_{t}-A \cap U_{t}\right) / C^{*}$. On the other hand, $[P]$ is of rank $\leqq s-1$ by Proposition 3.1. Therefore, Lemma 3.4 implies that the number of possible cases of $\left(\operatorname{ord}_{A_{t}} h_{1}, \cdots, \operatorname{ord}_{A_{t}} h_{p}\right)$ is bounded by a constant depending only on $p$ and $s$. On the other hand, two members $h, h^{\prime}$ in $H_{\tilde{X}}^{*}(X)$ satisfy the condition $[h]=\left[h^{\prime}\right]$ if and only if $\operatorname{ord}_{A_{t}} h=\operatorname{ord}_{A_{t}} h^{\prime}$ for each $t$. From these facts, we conclude Theorem 3.2.

\section{A finiteness theorem of meromorphic maps into $P^{N}(C)$.}

Let $f$ be a meromorphic map of a complex space $X$ into $P^{N}(\boldsymbol{C})$.

DEFINITION 4.1. We say $f$ to be linearly nondegenerate if $f(X)$ is not included in any hyperplane in $P^{N}(C)$.

The purpose of this section is to prove the following

THEOREM 4.2. Let $X$ be a complex space such that $X=\tilde{X}-A$ for a compact complex space $\tilde{X}$ and a thin analytic subset $A$ of $\tilde{X}$. For hyperplanes $H_{1}, \cdots, H_{N+2}$ on $P^{N}(C)$ located in general position and effective divisors $E_{1}, \cdots, E_{N+2}$ on $X$, consider the set $\mathscr{F}$ of all linearly nondegenerate meromorphic maps of $X$ into $P^{N}(\boldsymbol{C})$ such that $f^{*} H_{\imath}=E_{\imath}(1 \leqq i \leqq N+2)$. Then, $\# \mathscr{F}$ is bounded by a constant depending only on $N$ and the number of irreducible components of $A$.

For the proof, we need some preparations. We first recall the following generalization of the classical Picard-Borel theorem, which was proved by the author in [4] and by M.L. Green in [8] independently.

Proposition 4.3. Let $U^{n}=\left\{\left(z_{1}, \cdots, z_{n}\right) ;\left|z_{\imath}\right|<1\right\}, A:=U^{n} \cap\left\{z_{1}=0\right\}$ and let $f_{1}, \cdots, f_{p}$ be nowhere zero holomorphic functions on $U^{n}-A$. If each $f_{2} / f_{j}(i \neq j)$ has essential singularities along $A$, then $f_{1}, \cdots, f_{p}$ are linearly independent over the field $M\left(U^{n}\right)$ of all meromorphic functions on $U^{n}$.

For the proof, see [4], p. 280. 
We obtain from this the following:

Proposition 4.4. Let $\alpha^{1}, \cdots, \alpha^{p} \in M(\tilde{X})^{*}$ and $f_{1}, \cdots, f_{p} \in H^{*}(X)$ satisfying the condition

$$
\alpha^{1} f_{1}+\cdots+\alpha^{p} f_{p}=0
$$

Consider a partition of indices

$$
\{1,2, \cdots, p\}=J_{1} \cup J_{2} \cup \cdots \cup J_{k}
$$

such that $i$ and $j$ are contained in the same class $J_{l}$ if and only if $f_{2} / f_{3}$ has a meromorphic extension to $\tilde{X}$. Then, $\sum_{\imath \in J} \alpha^{2} f_{\imath}=0$ for each $l=1,2, \cdots, k$.

Proof. This is shown by induction on $k$. We have nothing to prove for the case $k=1$. Assume that $k \geqq 2$ and Proposition 4.3 holds for the case $\leqq k-1$. Then some $f_{\imath_{0}} / f_{j_{0}}\left(i_{0} \neq j_{0}\right)$ has an essential singularity and so essential singularities at all points of an irreducible component $A_{t}$ of $A$. Take a point $x_{0} \in R\left(A_{t}\right)$ and choose holomorphic local coordinates $z_{1}, \cdots, z_{n}$ on a neighborhood $U^{n}$ of $x_{0}$ in $\tilde{X}$ such that $x_{0}=(0), U^{n}=\left\{\left|z_{\imath}\right|<1\right\}$ and $U^{n} \cap A=U^{n} \cap\left\{z_{1}=0\right\}$. Let

$$
\{1, \cdots, p\}=J_{1}^{\prime} \cup \cdots \cup J_{k^{\prime}}^{\prime}
$$

be a partition such that $i$ and $j$ are in the same class $J_{m}^{\prime}$ if and only if $f_{i} / f_{j}$ has a meromorphic extension to $U^{n}$. Then, we see $k^{\prime} \geqq 2$ and each $J_{l}$ is included in some $J_{m}^{\prime}$. Changing indices, we may assume $m \in J_{m}^{\prime}$ for $1 \leqq m \leqq k^{\prime}$. Set

$$
\beta^{m}:=\sum_{\imath \in J_{m}^{\prime}} \alpha_{i}\left(f_{i} / f_{m}\right) \quad\left(\in M\left(U^{n}\right)\right)
$$

for each $m$. Apply Proposition 4.3 to the identity

$$
\sum_{1 \leqq m \leqq k^{\prime}} \beta^{m} f_{m}=\sum_{1 \leqq \imath \leqq p} \alpha_{\imath} f_{\imath}=0
$$

to show $\beta^{m}=0$ on $U^{n}$ for each $m$. This concludes

$$
\sum_{I_{l} \subset J_{m}^{\prime}}\left(\sum_{\imath \in I_{l}} \alpha_{\imath} f_{\imath}\right)=\sum_{i \in J_{m}^{\prime}} \alpha_{\imath} f_{\imath}=0
$$

on $X$ for each $m$. Since $\#\left\{l ; I_{l} \subset J_{m}^{\prime}\right\}<k$, we have $\sum_{i \in I} \alpha_{\imath} f_{2}=0$ for each $l$ by the induction hypothesis. This completes the proof.

COROLlaRY 4.5. In the same situation as in Proposition 4.4, functions

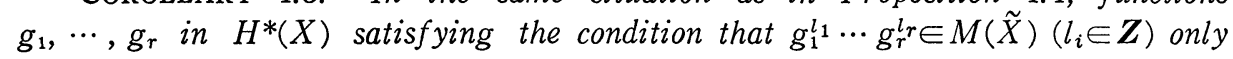
when $l_{1}=\cdots=l_{r}=0$ are algebraically independent over $M\left(U^{n}\right)$.

Proof. Set $f_{\iota}:=g_{1}^{l_{1}} \cdots g_{r}^{l_{r}}$ for $\boldsymbol{l}=\left(l_{1}, \cdots, l_{r}\right)$. By the assumption, $f_{l} / f_{m} \notin M(\tilde{X})$ for any distinct $\boldsymbol{l}$ and $\boldsymbol{m}$. By proposition 4.4, there is no non-trivial linear relation with coefficients in $M^{*}(\tilde{X})$ among $\left\{f_{l}\right\}$. This shows Corollary 4.5. 
We next consider $p \times q$ matrices $\left(h_{\imath \jmath} ; 1 \leqq i \leqq p, 1 \leqq j \leqq q\right)$ with components $h_{\imath}$ in $H^{*}(X)$ for various $p$ and $q$.

Proposition 4.6. For each $q_{0}(\geqq 1)$ there exists some constant $Q\left(p, q_{0}\right)$ depending only on $p$ and $q_{0}$ such that, if $q>Q\left(p, q_{0}\right)$ and

$$
\operatorname{det}\left(h_{\imath} ; i=1, \cdots, p, j=j_{1}, \cdots, j_{p}\right)=0
$$

for all $j_{l}$ with $1 \leqq j_{l} \leqq q$, then there exist $r$ functions $k_{1}, \cdots, k_{r} \in H^{*}(X)$ with $2 \leqq r \leqq p$ such that, after a suitable change of indices if necessary, $\gamma_{\imath j}:=h_{\imath j} /\left(h_{1 j} k_{\imath}\right)$ $\in M(\tilde{X})$ for $1 \leqq i \leqq r, 1 \leqq j \leqq q_{0}$ and

$$
\operatorname{det}\left(\gamma_{\imath} ; i=1, \cdots, r, j=j_{1}, \cdots, j_{r}\right)=0
$$

for all $j_{l}$ with $1 \leqq j_{l} \leqq q_{0}$.

Proof. We consider the factor group $G=H^{*}(X) / H_{\tilde{X}}^{*}(X)$ which is obviously torsion free. Choose $\eta_{1}, \cdots, \eta_{t} \in H^{*}(X)$ such that $\left[\eta_{1}\right], \cdots,\left[\eta_{t}\right]$ are multiplicatively independent over $\boldsymbol{Z}$ and each $h_{\imath}$ is represented as

$$
h_{\imath \jmath}=\alpha_{\imath j} \eta_{1}^{\imath_{\imath \jmath}^{1}} \ldots \eta_{t}^{l_{\imath \jmath}^{t}} \quad(1 \leqq i \leqq p, 1 \leqq j \leqq q)
$$

for some $\alpha_{\imath j} \in H_{\tilde{X}}^{*}(X)$. Set $\boldsymbol{l}_{\imath j}=\left(l_{\imath \jmath}^{1}, \cdots, l_{\imath j}^{t}\right) \in \boldsymbol{Z}^{t}$ and take integers $p_{1}, \cdots, p_{t}, q_{\jmath}$ such that

$$
l_{\imath \jmath}:=l_{\imath j}^{1} p_{1}+\cdots+l_{\imath j}^{t} p_{t}+q_{j} \geqq 0,
$$

and $\boldsymbol{l}_{\imath j}-\boldsymbol{l}_{i^{\prime} j}=\boldsymbol{l}_{\imath j^{\prime}}-\boldsymbol{l}_{i^{\prime} j^{\prime}}$ if and only if $l_{\imath j}-l_{i^{\prime} j}=l_{2 j^{\prime}}-l_{i^{\prime} j^{\prime}}$ for $1 \leqq i, i^{\prime} \leqq p, 1 \leqq j$, $j^{\prime} \leqq q$, and minors

$$
A_{J}^{I}\left(\eta_{1}, \cdots, \eta_{t}\right):=\operatorname{det}\left(\alpha_{\imath j} \eta_{1}^{\imath_{i j}^{1}} \cdots \eta_{t}^{l_{i j}^{t}} ; i=i_{1}, \cdots, i_{s}, j=j_{1}, \cdots, j_{s}\right)
$$

satisfy the condition that $A_{J}^{I}\left(\eta_{1}, \cdots, \eta_{t}\right) \neq 0$ if and only if $A_{J}^{I}\left(u^{p_{1}}, \cdots, u^{p_{t}}\right) \neq 0$ for any $I=\left(i_{1}, \cdots, i_{s}\right)$ and $J=\left(j_{1}, \cdots, j_{s}\right)$. Set $P_{\imath j}(u):=\alpha_{i j} u^{l_{2 j}} \in M(\tilde{X})[u]$, where $M(\tilde{X})[u]$ denotes the ring of all polynomials in $u$ with coefficients in $M(\tilde{X})$. Then, we have

$$
\operatorname{rank}\left(P_{\imath j}(u) ; 1 \leqq i \leqq p, 1 \leqq j \leqq q\right)<p .
$$

In fact, by the assumption, we see

$$
\operatorname{det}\left(\alpha_{\imath j} \eta_{1}^{l_{\imath \jmath}^{1}} \ldots \eta_{t}^{l_{\imath \jmath}^{t}} \eta_{t+1}^{q_{j}} ; i=1, \cdots, p, j=j_{1}, \cdots, j_{p}\right)=0
$$

for all $\left(i_{l}\right)$, where $\eta_{t+1}$ is an arbitrary function in $H^{*}(X)$. This is an identity of rational functions with coefficients in $M(\tilde{X})$ and indeterminates $\eta_{1}, \cdots, \eta_{t+1}$ by Corollary 4.5. By substituting $\eta_{2}=u^{p_{i}}(1 \leqq i \leqq t)$ and $\eta_{t+1}=u$, we get (4.8).

We now apply Main Lemma in the previous paper [6], $\$ 2$, p. 531 , which remains valid if we replace the coefficient field $C$ by $M(\tilde{X})$. We can conclude that for each $q_{0}(\geqq 1)$ there exists some constant $Q\left(p, q_{0}\right)\left(>q_{0}\right)$ depending only on $p$ and $q_{0}$ such that, if $q>Q\left(p, q_{0}\right)$, then 


$$
l_{i 1}-l_{i^{\prime} 1}=l_{i 2}-l_{i^{\prime} 2}=\cdots l_{2 q_{0}}-l_{i^{\prime} q_{0}}
$$

for all $i, i^{\prime}$ with $1 \leqq i, i^{\prime} \leqq r$ and

$$
\operatorname{rank}\left(P_{\imath j}(u) ; 1 \leqq i \leqq r, 1 \leqq j \leqq q_{0}\right)<r
$$

after a suitable change of indices $i$ and $j$, where $2 \leqq r \leqq p$. Then we have

$$
\boldsymbol{l}_{i 1}-\boldsymbol{l}_{i^{\prime} 1}=\cdots=\boldsymbol{l}_{\imath q_{0}}-\boldsymbol{l}_{i^{\prime} q_{0}} .
$$

Set $\left(m_{12}, \cdots, m_{t 2}\right):=\boldsymbol{l}_{i 1}-\boldsymbol{l}_{11}$ and define $k_{\imath}=\eta_{2}^{m_{12}} \cdots \eta_{t}^{m_{t 2}}$, which satisfy the desired condition. This completes the proof of Proposition 4.6.

Next, we study functions $\lambda_{1}, \cdots, \lambda_{p}$ in $M(\tilde{X})^{*}$ and $p \times q$ matrices $\left(\gamma_{\imath}\right.$; $1 \leqq i \leqq p, 1 \leqq j \leqq q)$ with components in $H_{\tilde{X}}^{*}(X)$ such that

$$
\lambda_{1} \gamma_{1 j}+\cdots+\lambda_{p} \gamma_{p j}=0 \quad(1 \leqq j \leqq q)
$$

for various $p$ and $q$.

LEMMA 4.9. For each $q_{0}(\geqq 1)$ there exists a constant $Q^{\prime}\left(p, q_{0}\right)$ such that, if $q>Q^{\prime}\left(p, q_{0}\right)$, then there is some $s_{0}$ with $2 \leqq s_{0} \leqq p$ such that, after a suitable change of indices $i$ and $j$

$$
\lambda_{1} \gamma_{1 j}+\cdots+\lambda_{s_{0}} \gamma_{s_{0} j}=0
$$

and $\sum_{i \in I} \lambda_{2} \gamma_{\imath j} \neq 0$ for any $I \sqsubseteq\left\{1, \cdots, s_{0}\right\}$ and $1 \leqq j \leqq q_{0}$.

Proof. Set $q_{1}^{*}=0$ and define

$$
q_{l}^{*}:=\sum_{1 \leqq s \leqq l-1} q_{s}^{*} C_{s}+q_{0}
$$

inductively. We shall show that $Q\left(p, q_{0}\right)=q_{p}^{*}$ satisfies the desired condition. Suppose that $q>Q^{\prime}\left(p, q_{0}\right)$. For each $\iota=\left(i_{1}, \cdots, i_{s}\right)$ with $1 \leqq i_{1}<\cdots<i_{s} \leqq p$ $(2 \leqq s \leqq p)$ we set

$$
I_{\iota}=I_{\imath_{1} \cdots \imath_{s}}:=\left\{J ; \lambda_{\imath_{1}} \gamma_{\imath_{1} j}+\cdots+\lambda_{\imath_{s}} \gamma_{\imath_{s} j}=0\right\} .
$$

Take the smallest $s_{0}$ with $2 \leqq s_{0} \leqq p$ such that $\# I_{\iota}>q_{s_{0}}^{*}$ for some $\iota=\left(i_{1}, \cdots, i_{s_{0}}\right)$. We note here $\# I_{12 \ldots p}=q>q_{p}^{*}$. Choose some $\left(i_{1}, \cdots, i_{s_{0}}\right)$ with this property. By changing indices, we assume $i_{1}=1, \cdots, i_{s_{0}}=s_{0}$. Then, if $s<s_{0}$, we have $\sharp I_{\iota} \leqq q_{s}^{*}$ for any $\iota=\left(i_{1}, \cdots, i_{s}\right)$ with $1 \leqq i_{1}<\cdots<i_{s} \leqq s_{0}$. Therefore,

$$
\begin{aligned}
\#\left(\cup\left\{I_{\iota} ; \iota=\left(i_{1}, \cdots, i_{s}\right), 1 \leqq i_{1}<\cdots<i_{s} \leqq s_{0}, 2 \leqq s<s_{0}\right\}\right) \\
\quad \leqq \sum_{1 \leqq s \leqq s_{0}-1} q_{s s_{0}}^{*} C_{s} \\
\quad \leqq \sum_{1 \leqq s \leqq s_{0}-1} q_{s p}^{*} C_{s}=q_{s_{0}}^{*}-q_{0} .
\end{aligned}
$$

This implies that 


$$
\begin{gathered}
\#\left(I_{12 \cdots s_{0}}-\cup\left\{I_{\imath_{1} \cdots \imath_{s}} ; 1 \leqq i_{1}<\cdots<i_{s} \leqq s_{0}, 2 \leqq s<s_{0}\right\}\right) \\
>q_{s_{0}}^{*}-\left(q_{s_{0}}^{*}-q_{0}\right)=q_{0} .
\end{gathered}
$$

By changing indices, we can assume that $I_{12 \ldots s_{0}} \supset\left\{1,2, \cdots, q_{0}\right\}$ and $I_{\imath_{1} \ldots \nu_{s}} \cap$ $\left\{1,2, \cdots, q_{0}\right\}=\varnothing$ for any $\left(i_{1}, \cdots, i_{s}\right)$ with $2 \leqq s<s_{0}$. This shows Lemma 4.9.

We now start to prove Theorem 4.2. We may identify $P^{N}(\boldsymbol{C})$ with the subspace

$$
H_{0}:=\left\{\left(w_{1}: \cdots: w_{N+2}\right) ; w_{1}+\cdots+w_{N+2}=0\right\}
$$

of $P^{N+1}(\boldsymbol{C})$ and $H_{2}$ with $H_{0} \cap\left\{w_{\imath}=0\right\}(1 \leqq i \leqq N+2)$, where $\left(w_{1}: \cdots: w_{N+2}\right)$ is a system of homogeneous coordinates on $P^{N+1}(C)$. For convenience sake, we set $p=N+2$ in the following.

Assume that $\mathscr{F}$ contains $q$ distinct maps $f_{1}, \cdots, f_{q}$. We shall prove that $q$ is not larger than a constant $Q^{*}\left(p, s_{0}\right)$ depending only on $p$ and the number $s_{0}$ of irreducible components of $A$. Each $f$, can be represented as

$$
f_{j}=\left(\varphi_{1}, \cdots: \varphi_{p j}\right)
$$

with meromorphic functions $\varphi_{i j}$ on $X$ satisfying the condition

$$
\varphi_{1 j}+\cdots+\varphi_{p j}=0
$$

where we may assume $\varphi_{p j}=1$ by (2.1). By the assumption, $\varphi_{i j}(1 \leqq i \leqq p-1)$ are linearly independent over $\boldsymbol{C}$. Moreover, since $D_{\varphi_{i}}=f^{*} H_{i}-f^{*} H_{p}=E_{i}-E_{p}$ for every $j$, we see $h_{\imath \jmath}:=\varphi_{i j} / \varphi_{i 1} \in H^{*}(X)$. We then have

$$
\varphi_{11} h_{1 j}+\cdots+\varphi_{p 1} h_{p_{j}}=0
$$

for $1 \leqq j \leqq q$. Therefore, $h_{\imath}(1 \leqq i \leqq p, 1 \leqq j \leqq q)$ satisfy the assumption of Proposition 4.6.

Assume that $q_{1}$ mappings among the maps $f_{\jmath}$, say $f_{1}, \cdots, f_{q_{1}}$, have meromorphic extensions to $\tilde{X}$. Then, for $j=1, \cdots, q_{1}, h_{\imath j} \in H_{\tilde{X}}^{*}(X), \quad \sum_{1 \leqq \imath s p} \varphi_{i 1} h_{\imath j}=0$ and $\sum_{i \in I} \varphi_{i 1} h_{\imath j} \neq 0$ whenever $I \subsetneq\{1,2, \cdots, p\}$. Therefore, we can apply Theorem 3.2 to these functions to show that the number of the distinct systems $\left(\left[h_{1 j}\right], \cdots,\left[h_{p j}\right]\right)\left(1 \leqq j \leqq q_{1}\right)$ is bounded by a constant $Q^{*}\left(p, s_{0}\right)$ depending only on $p$ and $s_{0}$. On the other hand, if

$$
\left(\left[h_{1 j}\right], \cdots,\left[h_{p j}\right]\right)=\left(\left[h_{1 j^{\prime}}\right], \cdots,\left[h_{p j^{\prime}}\right]\right)
$$

for some $j, j^{\prime}$, then we can write $\varphi_{i j^{\prime}}=c_{i} \varphi_{i}$, for some $c_{i} \in C^{*}$. In this case, we have $c_{1} \varphi_{1 j}+\cdots+c_{p} \varphi_{p j}=\varphi_{1 j^{\prime}}+\cdots+\varphi_{p^{\prime}}=0$. Since $\varphi_{1 \jmath}, \cdots, \varphi_{p-1}$ are linearly independent over $\boldsymbol{C}$, we get $c_{1}=\cdots=c_{p}$ and so $j=j^{\prime}$. This concludes $q_{1} \leqq$ $Q^{*}\left(p, s_{0}\right)$.

For our purpose, by the above shown fact we may assume that every $f$, $(1 \leqq j \leqq q)$ has essential singularities along $A$. For the case $p=3$, it suffices to take $Q^{*}\left(p, s_{0}\right)=Q(3,2)$, where $Q\left(p, q_{0}\right)$ is the quantity given in Proposition 4.6. 
In fact, if $q>Q(3,2)$, then after a suitable change of indices we have

$$
\gamma_{\imath \jmath}:=h_{\imath j} /\left(h_{1 j} k_{\imath}\right) \in M(\tilde{X})
$$

for some $k_{1}, \cdots, k_{r} \in H^{*}(X)$ and $\operatorname{rank}\left(\gamma_{\imath} ; \imath=1,2,3, j=1,2\right)<r$, where $2 \leqq r \leqq 3$. In the case where $\operatorname{det}\left(\gamma_{i j} ; 1 \leqq i, j \leqq 2\right)=0$, there exists some $\gamma$ in $M(\tilde{X})^{*}$ with $\gamma_{12}=\gamma \gamma_{11}, \gamma_{22}=\gamma \gamma_{21}$. Then, $\varphi_{i 2}=h_{i 2} \varphi_{i 1}=\gamma_{i 2} h_{12} k_{i} \varphi_{i 1}=\gamma \gamma_{i 1} h_{12} \varphi_{i 1} k_{2}=\gamma h_{12} \varphi_{i 1} \quad$ for $i=1,2$, and $\varphi_{32}=-\left(\varphi_{12}+\varphi_{22}\right)=\gamma h_{12} \varphi_{31}$. So, $f_{1}=f_{2}$. This is a contradiction. In the case where $\operatorname{det}\left(\gamma_{i j} ; 1 \leqq i, j \leqq 2\right) \neq 0$, we have necessarily $r=3$ and the identities

$$
\varphi_{11} k_{1} \gamma_{1 j}+\varphi_{21} k_{2} \gamma_{2 j}+\varphi_{31} k_{3} \gamma_{3 j}=0 \quad(j=1,2)
$$

imply that $\left(\varphi_{i 1} k_{\imath}\right) /\left(\varphi_{11} k_{1}\right) \in M(\tilde{X})$ for $i=2,3$. This concludes that $f_{2}$ has a meromorphic extension to $\tilde{X}$, which contradicts the assumption.

Assume that there exist $Q^{*}\left(3, s_{0}\right), \cdots, Q^{*}\left(p-1, s_{0}\right)$ with the desired properties for each $s_{0}$, where $Q^{*}\left(l-1, s_{0}\right)<Q^{*}\left(l, s_{0}\right)$ for $l=4, \cdots, p-1$. Let $R\left(p, s_{0}\right)$, $Q\left(p, q_{0}\right)$ and $Q^{\prime}\left(p, q_{0}\right)$ be the quantities given by Theorem 3.2, Proposition 4.6 and Lemma 4.9 respectively, where we may assume $R\left(p-1, s_{0}\right) \leqq R\left(p, s_{0}\right)$. We now define inductively the numbers $Q^{(l)}\left(p, s_{0}\right)$ for $l=1,2$ and $Q^{*}\left(p, s_{0}\right)$ by the following conditions;

(4.11) $Q^{(1)}\left(p, s_{0}\right)>R\left(p, s_{0}\right)\left(Q^{*}\left(p-1, s_{0}\right)+1\right)$

(4.12) $Q^{(2)}\left(p, s_{0}\right)>Q^{\prime}\left(p, Q^{(1)}\left(p, s_{0}\right)+1\right)$,

(4.13) $Q^{*}\left(p, s_{0}\right) \geqq Q\left(p, Q^{(2)}\left(p, s_{0}\right)\right)$,

(4.14) $Q^{(l)}\left(p, s_{0}\right) \geqq Q^{(l)}\left(p-1, s_{0}\right)$ for each $l=1,2$ and $Q^{*}\left(p, s_{0}\right) \geqq Q^{*}\left(p-1, s_{0}\right)$.

Suppose that $q>Q^{*}\left(p, s_{0}\right)$. Then, by the use of Proposition 4.6 and (4.13), after a suitable change of indices we can find some $k_{1}, \cdots, k_{r} \in H^{*}(X)(2 \leqq r \leqq p)$ such that $\gamma_{\imath j}=h_{\imath j} /\left(h_{1 j} k_{\imath}\right) \in M(\tilde{X})$ for $1 \leqq i \leqq r$ and $1 \leqq j \leqq Q^{(2)}\left(p, s_{0}\right)$ and

$$
\operatorname{rank}\left(\gamma_{\imath \jmath} ; 1 \leqq \imath \leqq r, 1 \leqq j \leqq Q^{(2)}\left(p, s_{0}\right)\right)<r .
$$

Therefore, there exists some $\lambda_{1}, \cdots, \lambda_{r} \in M(\tilde{X})$ with $\left(\lambda_{1}, \cdots, \lambda_{r}\right) \neq(0, \cdots, 0)$ such that

$$
\lambda_{1} \gamma_{1 j}+\cdots+\lambda_{r} \gamma_{r j}=0 \quad\left(1 \leqq j \leqq Q^{(2)}\left(p, s_{0}\right)\right) .
$$

Changing indices if necessary, we may assume that $\lambda_{1} \neq 0, \cdots, \lambda_{u} \neq 0, \lambda_{u+1}=\cdots$ $=\lambda_{r}=0$. Then, by the use of Lemma 4.9 , we can assume that

$$
\lambda_{1} \gamma_{1 j}+\cdots+\lambda_{u} \gamma_{u j}=0
$$

for any $j=1,2, \cdots, Q^{(1)}\left(p, s_{0}\right)+1$ and $\sum_{i \in I} \lambda_{2} \gamma_{\imath \jmath} \neq 0$ for $I \subsetneq\{1,2, \cdots, u\}$. Apply Theorem 3.2 to the functions $\alpha_{1}=\lambda_{1}, \cdots, \alpha_{u}=\lambda_{u}$ to show that the number of distinct systems among

$$
\left.\left\{\left(\left[\gamma_{1}\right], \cdots,\left[\gamma_{u}\right]\right]\right) \in \oplus^{u}\left(H_{\tilde{X}}^{*}(X) / C^{*}\right) ; 1 \leqq j \leqq Q^{(1)}\left(p, s_{0}\right)\right\}
$$

is at most $R\left(u, s_{0}\right)\left(\leqq R\left(p, s_{0}\right)\right)$. Among $Q^{(1)}\left(p, s_{0}\right)$ systems $\left(\gamma_{1 j}, \cdots, \gamma_{u j}\right)$ which belongs to the same class $\left(\left[\gamma_{1}\right], \cdots,\left[\gamma_{u j}\right]\right)$. Therefore, after changing indices and renewing $\varphi_{i j}$, we can write 


$$
f_{j}=\left(c_{1 j} k_{1}^{*}: \cdots: c_{u j} k_{u}^{*}: \varphi_{u+1}: \cdots: \varphi_{p_{j}}\right)
$$

with some $c_{\imath j} \in C^{*}$ and $k_{1}^{*}, \cdots, k_{u}^{*} \in H^{*}(X)$ for $j=1,2, \cdots, Q^{*}\left(p-1, s_{0}\right)$. Then by (4.15) we see

$$
\operatorname{rank}\left(c_{\imath \jmath} ; 1 \leqq i \leqq u, 1 \leqq \jmath \leqq Q^{*}\left(p-1, s_{0}\right)+1\right)<u .
$$

We may write

$$
c_{1 j}=\sum_{2 \leqq \imath \unlhd u} c_{\imath j} d_{\imath}
$$

for some $d_{i} \in \boldsymbol{C}(2 \leqq i \leqq u)$. Set $k_{i}^{* *}:=k_{i}^{*}+d_{i} k_{1}^{*}$ for $2 \leqq i \leqq u$ and define the maps

$$
\tilde{f}_{j}=\left(c_{2 j} k_{2}^{* *}: \cdots: c_{u j} k_{u}^{* *}: \varphi_{u+1}: \cdots: \varphi_{p-1 j}\right)
$$

of $X$ into $P^{N-1}(\boldsymbol{C})$ for $j=1,2, \cdots, Q^{*}\left(p-1, s_{0}\right)+1$. Then $\tilde{f}_{j}$ are all nondegenerate. For $k_{1}^{*}, \cdots, k_{u}^{*}, \varphi_{u+1,}, \cdots, \varphi_{p-1}$ are linearly independent by the assumption and so $k_{2}^{*}+d_{2} k_{1}^{*}, \cdots, k_{u}^{*}+d_{u} k_{1}^{*}, \varphi_{u+1}, \cdots, \varphi_{p-1}$ are also linearly independent. Moreover, if

$$
\begin{aligned}
\left(c_{2 j} k_{2}^{* *}: \cdots: c_{u j} k_{u}^{* *}: \varphi_{u+1 \jmath}: \cdots: \varphi_{p-1 j}\right) \\
\quad=\left(c_{2 j^{\prime}} k_{2}^{* *}: \cdots: c_{u j^{\prime}} k_{u}^{* *}: \varphi_{u+1 j^{\prime}}: \cdots: \varphi_{p-1 j^{\prime}}\right),
\end{aligned}
$$

then $c_{\imath \jmath}=d c_{\imath j^{\prime}}(2 \leqq i \leqq u)$ for some $d \in C^{*}$ and

$$
\begin{aligned}
c_{1 j} k_{1}^{*} & =-\left(c_{2 j} k_{2}^{*}+\cdots+c_{u j} k_{u}^{*}+\varphi_{u+1 j}+\cdots+\varphi_{p j}\right) \\
& =-d\left(c_{2 j^{\prime}} k_{2}^{*}+\cdots+c_{u j^{\prime}} k_{u}^{*}+\varphi_{u+1 j^{\prime}}+\cdots+\varphi_{p j^{\prime}}\right) \\
& =d c_{1 j^{\prime}} k_{1}^{*},
\end{aligned}
$$

which implies $f_{j}=f_{j^{\prime}}$. Therefore, the set $\mathscr{F}^{\prime}$ of all meromorphic maps $\tilde{f}$ of $X$ into $P^{N-1}(\boldsymbol{C})=P^{N}(\boldsymbol{C}) \cap\left\{w_{1}=0\right\}$ with $\tilde{f}^{*} H_{\imath}=D_{k_{i}^{* *}}(2 \leqq i \leqq u)$ and $\tilde{f}^{*} H_{\imath}=E_{\imath}$ $(u+1 \leqq i \leqq p)$ contains $Q^{*}\left(p-1, s_{0}\right)+1$ distinct elements. This contradicts the induction hypothesis. The proof of Theorem 4.2 is completed.

\section{Proof of Main Theorem.}

For the proof of Main Theorem, we need some lemmas.

LEMMA 5.1 ([1]). Let $L \rightarrow Y$ be a very ample line bundle over an $N$-dimensional smooth projective algebraic manifold $Y$ and $\varphi_{1}, \cdots, \varphi_{N+1} \in H^{0}(Y, \mathcal{O}(L))^{*}$. If

$$
\bigcap_{1 \leqq j \leqq N+1} \operatorname{Supp} D_{\varphi_{j}}=\varnothing \text {, }
$$

then $\varphi_{1} / \varphi_{N+1}, \cdots, \varphi_{N} / \varphi_{N+1}$ are algebraically independent over $\boldsymbol{C}$.

For the proof, see [1], p. 213. 
LEMMA 5.2 ([1]). Let $L \rightarrow Y$ be a line bundle as in Lemma 5.1 and $\varphi_{1}, \cdots, \varphi_{N+2} \in H^{0}(Y, O(L))^{*}$ satisfy the condition that

$$
\operatorname{Supp} D_{\varphi_{1}} \cap \cdots \cap \operatorname{Supp} D_{\varphi_{J-1}} \cap \operatorname{Supp} D_{\varphi_{j+1}} \cap \cdots \cap \operatorname{Supp} D_{N+2}=\varnothing
$$

for each $j=1, \cdots, N+2$. Take a nonzero irreducible homogeneous polynomial $R\left(u_{1}, \cdots, u_{N+2}\right)$ such that $R\left(\varphi_{1}, \cdots, \varphi_{N+2}\right)=0$ on $Y$, and set

$$
R(u)=\sum_{\imath_{1}+\cdots+\imath_{N+2}=k} a_{\imath_{1} \cdots \imath_{N+2}} u_{1}^{\imath_{1}} \cdots u_{N+2}^{\imath_{N+2}} .
$$

Then,

$$
a_{k 0 \ldots 0} \neq 0, a_{0 k 0 \ldots 0} \neq 0, \cdots, a_{00 \ldots 0 k} \neq 0 .
$$

For the proof, see [1], pp. 213 216.

LEMMA 5.3. Let $L$ be a line bundle over an $N$-dimensional compact complex manifold $Y$ which has at least one system of $N+1$ algebraically independent holomorphic sections. Then, there exists a positive constant $k_{L}$ depending only on $L$ such that for arbitrary algebraically independent $\varphi_{1}, \cdots, \varphi_{N+1} \in H^{0}(Y, \mathcal{O}(L))$ the meromorphic map $\Phi:=\left(\varphi_{1}: \cdots: \varphi_{N+1}\right): Y \rightarrow P^{N}(C)$ satisfies the condition that $\# \Phi^{-1} \Phi(w) \leqq k_{L}$ for every point $w$ in a nonempty Zariski open subset $G$ of $Y$.

For the proof, see [6], p. 537.

Now, we start to prove Main Theorem. By the assumption, there exists a positive integer $d$ such that $L^{d}$ is very ample. For our purpose, we may replace $L$ by $L^{d}$ and so assume that $L$ is very ample from the beginning. Indeed, the set $\mathcal{E}$ is included in the set of all meromorphic maps of $X$ into $Y$ which are algebraically nondegenerate with respect to $L$ and satisfy the condition $f^{*}\left(d D_{i}\right)=d E_{\imath}$. Moreover, the divisors $d D_{1}, \cdots, d D_{N+2} \in\left|L^{d}\right|$ satisfy the assumption of Main Theorem. Therefore, it suffices to prove Main Theorem for $L^{d}$.

Take holomorphic sections $\varphi_{1}, \cdots, \varphi_{N+2}$ of $L$ with $D_{i}=D_{\varphi_{i}}(1 \leqq i \leqq N+2)$. Then, $\varphi_{1} / \varphi_{N+2}, \cdots, \varphi_{N+1} / \varphi_{N+2}$ are algebraically dependent and $\varphi_{1} / \varphi_{N+1}, \cdots$, $\varphi_{N} / \varphi_{N+1}$ are algebraically independent by Lemma 5.1. It follows from these facts that there exists a nonzero homogeneous polynomial $R(u)$ of degree $k \geqq 1$ such that

We write

$$
R\left(\varphi_{1}, \cdots, \varphi_{N+2}\right)=0 \text {. }
$$

$$
R(u)=\sum_{1 \leq j \leq s+2} R_{j}(u),
$$

where $R_{j}(u)$ are nonzero monomials. By virtue of Lemma 5.2, we may assume

$$
R_{1}(u)=c_{1} u_{1}^{k}, \cdots, R_{N+2}(u)=c_{N+2} u_{N+2}^{k},
$$

where $c_{i} \in C^{*}(1 \leqq i \leqq N+2)$.

We now consider a holomorphic map $\Psi: Y \rightarrow P^{s}(\boldsymbol{C})$ defined by 


$$
\Psi(y)=\left(R_{1}\left(\varphi_{1}(y), \cdots, \varphi_{N+2}(y)\right): \cdots: R_{s+1}\left(\varphi_{1}(y), \cdots, \varphi_{N+2}(y)\right)\right) .
$$

Instead of the set $\mathcal{E}$ we study the set $\tilde{\mathcal{E}}$ of all meromorphic maps $\tilde{f}:=\Psi \cdot f$ of $X$ into $P^{s}(\boldsymbol{C})$ with $f \in \tilde{\mathcal{E}}$. Each $\tilde{f} \in \tilde{\mathcal{E}}$ is linearly nondegenerate because $f$ is algebraically nondegenerate with respect to $L$. We set

$$
\begin{aligned}
\tilde{H}_{j} & :=\left\{v_{j}=0\right\} \quad(1 \leqq j \leqq s+1) \\
\tilde{H}_{s+2} & :=\left\{v_{1}+\cdots+v_{s+1}=0\right\},
\end{aligned}
$$

where $\left(v_{1}: \cdots: v_{s+1}\right)$ denotes homogeneous coordinates on $P^{s}(\boldsymbol{C})$. Then, the hyperplanes $\widetilde{H}_{1}, \cdots, \widetilde{H}_{s+1}$ are located in general position. Moreover, we set

$$
\tilde{E}_{j}=l_{1} E_{1}+\cdots+l_{N+2} E_{N+2}
$$

if $R_{j}(u)=c u_{1}^{l_{1}} \cdots u_{N+2}^{l_{N+2}}\left(c \in C^{*}\right)$. We then have

$$
f^{*}\left(\tilde{H}_{j}\right)=f^{*}\left(\Psi^{*}\left(\tilde{H}_{\jmath}\right)\right)=\tilde{E}_{\jmath} \quad(1 \leqq j \leqq s+2) .
$$

As a consequence of Theorem 4.2 , we obtain $\sharp \tilde{E}<\infty$. Take an arbitrary map $f_{0} \in \mathcal{E}$. It suffices to show that

$$
\sharp\left\{f \in \mathcal{E} ; \Psi \cdot f=\Psi \cdot f_{0}\right\}<\infty .
$$

To see this, we apply Lemma 5.3 to algebraically independent sections $\left(\varphi_{1}\right)^{k}, \cdots,\left(\varphi_{N+1}\right)^{k}$. By the help of (5.4) we can conclude that there exists a positive constant $d_{0}$ such that $\sharp \Psi^{-1} \Psi(w) \leqq d_{0}$ for every point $w$ in a nonempty Zariski open subset $G$ of $Y$. Suppose that there are mutually distinct $q+1$ meromorphic maps $f_{0}, \cdots, f_{q} \in \mathcal{E}$ such that $\Psi \cdot f_{j}=\Psi \cdot f_{0}$. Set

$$
G^{*}:=\left\{x \in X ; f_{j}(x) \in G \text { for all } j \text { and } f_{j}(x) \neq f_{j^{\prime}}(x) \text { for } 0 \leqq j<j^{\prime} \leqq q\right\} .
$$

By the assumption of nondegeneracy of $f_{\imath}, G^{*}$ is an open dense subset of $X$. For a point $x_{0} \in G^{*}$ we have $f_{0}\left(x_{0}\right) \in G$ and

$$
\left\{f_{0}\left(x_{0}\right), \cdots, f_{q}\left(x_{0}\right)\right\} \subset \Psi^{-1} \Psi\left(x_{0}\right),
$$

whence $q+1 \leqq d_{0}$. This completes the proof of Main Theorem.

\section{REFERENCES}

[1] Y. Aihara AND S. MoRI, Algebraic degeneracy theorem for holomorphic mappings into smooth projective algebraic varieties, Nagoya Math. J., 84 (1981), 209-218.

[2] E. Borel, Sur les zéros des fonctions entières, Acta Math., 20 (1897), 357-396.

[3] H. CARTAN, Sur les systèmes de fonctions holomorphes à variété linéaires lacunaires et leurs applications, Ann. Sci. Ecole Norm. Sup., 45 (1928), 255-346.

[4] H. Fujimoto, On meromorphic maps into the complex projective space, J. Math. Soc. Japan, 26 (1974), 272-288.

[5] H. Fujimoto, Remarks to the uniqueness problem of meromorphic maps into $P^{N}(\boldsymbol{C})$, IV, Nagoya Math. J., 83 (1981), 153-181. 
[6] H. Fujimoto, On meromorphic maps into a compact complex manifold, J. Math. Soc. Japan, 34 (1982), 527-539.

[7] H. Fujimoto, A finiteness of meromorphic maps into a compact normal complex space, Sci. Rep. Kanazawa Univ., 30 (1985), 15-25.

[8] M.L. Green, Some Picard theorems for holomorphic maps to algebraic varieties, Amer. J. Math., 97 (1975), 43-75.

[9] K. Langmann, Anwendungen des Satzes von Picard, Math. Ann., 266 (1984), 369-390.

[10] K. Langmann, Picard-Borel-Eigenschafte und Anwendungen, Math. Z., 192 (1986), 587-601.

Department of Mathematics

Faculty of Science

KANAZAWA UNIVERSITY

Marunouchi, Kanazawa, 920

JAPAN 